\title{
SIMPLE CONTINUED FRACTIONS AND SPECIAL RELATIVITY
}

\author{
DOUGLAS HENSLEY ${ }^{1}$
}

\begin{abstract}
Let $E_{0}, E_{1}, \ldots, E_{n}$ be inertial frames of reference in a one dimensional relativistic universe where the speed of light is $c=\sqrt{k}, k$ some natural number. For $n>1$ let $E_{n}$ have velocity 1 with respect to $E_{n-1}$. Let $x_{n}$ denote the velocity of $E_{n}$ with respect to $E_{0}$. Then only if $k=2,3$ or 5 will $x_{n}$ be a simple continued fraction convergent of $\sqrt{k}$ infinitely often.
\end{abstract}

Introduction. Let $c$ denote the speed of light, and let $E_{0}, E_{1}$ and $E_{2}$ be inertial frames of reference with collinear velocities. Let the velocity of $E_{1}$ with respect to $E_{0}$ be $x$, and of $E_{2}$ with respect to $E_{1}, y$. The theory of special relativity then gives the velocity of $E_{2}$ with respect to $E_{0}$ as $(x+y) /(1+$ $x y c^{-2}$ ), which we denote $x \bigoplus_{c} y$.

Let units of measurement be chosen so that $c^{2}$ is an integer. Given $x_{0}$, let $x_{n}$ denote the sum $x_{0} \oplus_{c} 1 \oplus_{c} 1 \cdots \oplus_{c} 1$ ( $n$ additions of 1 ), so that $x_{n}=x_{n-1}$ $\oplus_{c} 1$ if $n \geqslant 1$. If $x_{0}$ is rational, the sequence $\left(x_{n}\right)$ will be a sequence of rational numbers converging to the square root of the integer $c^{2}$.

We ask whether $x_{n}$ is infinitely often a simple continued fraction convergent of $c$. K. B. Stolarsky noted that if $x_{0}=1$ and $c=\sqrt{2}$ or $\sqrt{3}$, this is the case, and posed the more general problem. First let us note one more example. If $c=\sqrt{5}$ and $x_{0}=1$ then $x_{6 n+2}$ is the $2 n$th convergent of $\sqrt{5}$. In what follows we show why there are no other examples. I wish to thank Professor Freeman Dyson for his helpful comments.

THEOREM. If $c^{2}=k$ is a nonsquare integer $>5$ and if $x_{0} \in Q$ then there are at most finitely many $n$ such that $x_{n}$ is a continued fraction convergent of $\sqrt{k}$.

Proof. Let $a=a_{0}+a_{1} \sqrt{k}$ be such that $a_{0}, a_{1} \in \mathbf{Z}$ and $x_{0}=\sqrt{k}(a-$ a) $/(a+\bar{a})$, where conjugation is in the field $Q(\sqrt{k})$. Let $\alpha=k+\sqrt{k}$.

LEMMA. If $x=\sqrt{k}(b-\bar{b}) /(b+\bar{b}), b \in Q(\sqrt{k})$, then $x \oplus_{c} 1=\sqrt{k}(b \alpha$ $-\bar{b} \bar{\alpha}) /(b \boldsymbol{\alpha}+\bar{b} \bar{\alpha})$.

Proof. Let $b=b_{0}+b_{1} \sqrt{k}$ with $b_{0}, b_{1} \in Q$. Then

$$
x \oplus_{c} 1=\left(b_{1} k / b_{0}+1\right) /\left(1+b_{1} / b_{0}\right)=\left(b_{1} k+b_{0}\right) /\left(b_{1}+b_{0}\right) .
$$

Received by the editors April 22, 1977.

AMS (MOS) subject classifications (1970). Primary 10A30, 10A35, 10F20, 83A05.

Key words and phrases. Special relativity, simple continued fraction, velocity addition laws.

'Supported in part by NSF grant MCS72-05055 A04. 
Likewise

$$
\begin{aligned}
\sqrt{k}(b \alpha-\bar{b} \bar{\alpha}) /(b \alpha+\bar{b} \bar{\alpha}) & =\sqrt{k}\left(\left(b_{1} k+b_{0}\right) \sqrt{k} /\left(b_{0} k+b_{1} k\right)\right) \\
& =\left(b_{1} k+b_{0}\right) /\left(b_{1}+b_{0}\right) .
\end{aligned}
$$

COROLlARY. If $x_{0}, a$ and $\alpha$ are as above, then

$$
x_{n}=\sqrt{k}\left(a \alpha^{n}-\bar{a} \bar{\alpha}^{n}\right) /\left(a \alpha^{n}+\bar{a} \bar{\alpha}^{n}\right) .
$$

Proof. Take $b=a \alpha^{n-1}$. Then $\bar{b}=\bar{a} \bar{\alpha}^{n-1}$ and the induction step follows from the Lemma above. And by assumption the claim holds when $n=0$.

Recall that for each nonsquare $k$ there exists an $M$ such that if $\left(d_{1}, d_{2}\right)=1$ and $d_{1} / d_{2}$ is a continued fraction convergent of $\sqrt{k}$, then $\left|d_{1}^{2}-k d_{2}^{2}\right|<M$ [1].

Now suppose that in $Q(\sqrt{k})$ there is some prime ideal $p$ which divides $\bar{\alpha} / \alpha$ to a positive power. This happens if and only if $\alpha / \bar{\alpha}$ is not an integer of $Q(\sqrt{k})$. If for some $n, x_{n}=\left(d_{1} / d_{2}\right)$ is a continued fraction convergent of $\sqrt{k}$ then by the Corollary,

$$
\sqrt{k}\left(a \alpha^{n}-\bar{a} \bar{\alpha}^{n}\right) /\left(a \alpha^{n}+\bar{a} \bar{\alpha}^{n}\right)=d_{1} / d_{2}
$$

so that

$$
k\left(a \alpha^{n}-\bar{a} \bar{\alpha}^{n}\right)^{2} d_{2}^{2}-\left(a \alpha^{n}+\bar{a} \bar{\alpha}^{n}\right)^{2} d_{1}^{2}=0,
$$

and

$$
k\left(a-\bar{a}(\bar{\alpha} / \alpha)^{n}\right)^{2} d_{2}^{2}-\left(a+\bar{a}(\bar{\alpha} / \alpha)^{n}\right)^{2} d_{1}^{2}=0 .
$$

Since $p^{n}$ divides $(\bar{\alpha} / \alpha)^{n}, p^{n}$ divides $a^{2}\left(k d_{2}^{2}-d_{1}^{2}\right)$. Since $k d_{2}^{2}-d_{1}^{2}$ is a nonzero integer with absolute value $<M$ it contains only finitely many powers of $p$. Therefore there can be only finitely many $n$ for which $x_{n}$ is a continued fraction convergent of $\sqrt{k}$.

To complete the proof we note that $\alpha / \bar{\alpha}$ is an integer of $Q(\sqrt{k})$ if and only if the polynomial

$$
(x-\alpha / \bar{\alpha})(x-\bar{\alpha} / \alpha)=x^{2}-2(k+1) x /(k-1)+1
$$

has integer coefficients, and that this occurs exactly when $k=2,3$ or 5 .

\section{BIBLIOGRAPHY}

1. I. Niven and H. S. Zuckerman, An introduction to the theory of numbers, 2nd ed., Wiley, New York, 1966, Theorems 7.19 and 7.22.

School of Mathematics, The Institute for Advanced Study, Princeton, New Jersey 08540

Current address: Department of Mathematics, Texas A \& M University, College Station, Texas 77843 